\title{
A class of shape preserving 5-point $n$-ary approximating schemes
}

\author{
Robina Bashira, Ghulam Mustafa, ${ }^{a, *}$, Praveen Agarwal ${ }^{b}$ \\ ${ }^{a}$ Department of Mathematics, The Islamia University of Bahawalpur, Bahawalpur, Pakistan. \\ ${ }^{b}$ Department of Mathematics, Anand International College of Engineering, Jaipur, India.
}

\begin{abstract}
A new class of shape preserving relaxed 5-point n-ary approximating subdivision schemes is presented. Further, the conditions on the initial data assuring monotonicity, convexity and concavity preservation of the limit functions are derived. Furthermore, some significant properties of ternary and quaternary subdivision schemes have been elaborated such as continuity, Hölder exponent, polynomial generation, polynomial reproduction, approximation order, and support of basic limit function. Moreover the visual performance of schemes has also been demonstrated through several examples.
\end{abstract}

Keywords: Approximating scheme, shape preserving, monotonicity, convexity, concavity, polynomial reproduction and generation.

2010 MSC: 68U05, 68U07, 65D17, 65D07, 65D05.

(C)2018 All rights reserved.

\section{Introduction}

The realization of the subdivision schemes for curve and surface designing imposes certain constraints on the computer based model. The model must accurately represent a variety of shapes, be amenable to analysis and manipulation, and must take into account the capabilities and limitations of both the computer and the designer. Initial work on approximating subdivision schemes was started by Rham [6]. Later on, Chaikin [4] introduced an algorithm to generate curve by a process of successively chopping off corners. In 1987, Dyn et al. [10] introduced the first work on linear interpolating schemes by using the approach of cubic Lagrange interpolating polynomial. In 1989, Deslauriers and Dubuc introduced generalized family of interpolating subdivision scheme based on Lagrange polynomial. Consequent to this, the research communities are interested in introducing higher arity schemes (i.e., ternary, quaternary and so on n-ary) which give better results and less computational cost. Mustafa and Khan [22] constructed a new 4-point quaternary approximating subdivision scheme having the higher smoothness and approximation order but smaller support than existing 4-point binary and ternary schemes. Mustafa and Rehman [24]

\footnotetext{
${ }^{*}$ Corresponding author

Email addresses: roba_bashir@hotmail.com (Robina Bashir), ghulam.mustafa@iub.edu.pk (Ghulam Mustafa),

goyal . praveen2011@gmail . com (Praveen Agarwal)
}

doi: $10.22436 /$ jmcs.018.03.11

Received: 2017-05-29 Revised: 2017-08-12 Accepted: 2017-08-25 
have presented general formulae for the mask of $(2 b+4)$-point $n$-ary interpolating and approximating schemes for any integer $b \geqslant 0$ and $n \geqslant 2$. Ghaffar et al. $[12,13]$ have presented an explicit formulae for the mask of 3-point and 4-point a-ary approximating subdivision schemes, respectively. Mustafa et al. [20] offered an explicit formula for the mask of odd points n-ary schemes.

The motivation of the present work is the desire to use two-steps techniques to construct a class of 5-point $n$-ary approximating subdivision schemes. This idea is firstly used by Lane and Riesenfeld [18]. They have presented two algorithms for parametric piecewise polynomial evaluation and generation. The mathematical development of these algorithms is shown to generalize to new algorithms for obtaining curve and surface intersections and for the computer display of parametric curves and surfaces. Augsdörfer et al. [1] first derived and analyzed families of variations on the four-point binary scheme, which improve on the scheme in various way which meet different requirements. These experiments demonstrate that manipulating the elementary highly local stages of each subdivision step is a useful mechanism for modifying the four-point scheme.

An essential tool to evaluate the significance of subdivision schemes is its shape preserving properties, which are regarded as geometrical properties of subdivision schemes. Dyn et al. [8] described the convexity in the useful sense and is realized for data fulfilling certain conditions in addition to the convexity conditions. Many subdivision schemes cannot preserve monotonicity and convexity in the present research field. Cai [2] introduced a four point interpolatory subdivision scheme which generates $C^{1}$ continuous curves in nonuniform control points and discussed the monotonicity preservation of the limit curve. Hassan et al. [15] presented an interpolating four-point ternary subdivision scheme, which is $\mathrm{C}^{2}$ continuous. The convexity preserving properties of the subdivision scheme (Hassan et al. 2002) has been discussed in Cai [3]. Hao et al. [14] introduced a linear 6-point binary approximating subdivision scheme which preserves convexity while its support is large. Tan et al. [31] presented only a binary four point subdivision scheme which preserve monotonicity and convexity of the limit curve. Owing to this we have evaluated monotonicity, convexity, and concavity preservation of our proposed ternary and quaternary schemes.

The paper is structured as follows. In Section 2, we introduce a two-steps algorithm which generates a class of 5-point $n$-ary approximating subdivision schemes. Smoothness analysis of proposed scheme is also presented in the same section. We discuss the conditions at the initial points guaranteeing monotonicity, convexity, and concavity preservation in Section 3 and numerical examples are also presented in this section. In Section 4, Hölder regularity, polynomial generation, polynomial reproduction, approximation order, and symmetry of basic limit function of ternary and quaternary subdivision schemes have been discussed. Conclusion is presented in Section 5.

\section{Algorithm for construction of schemes}

In this section, we present an algorithm for the construction of 5-point n-ary approximating subdivision schemes. This algorithm has two main steps. One step has been borrowed by 4 -point $n$-ary DD interpolating schemes [7]. That is during first step each segment of control polygon is divided into $n$-subsegments by inserting $n$ number of new points at position $1 / n, 2 / n, \ldots,(n-1) / n$ by 4 -point DDscheme. While the other step is to change the interpolating rule of DD-scheme by 5-point approximating rule.

Consider the open polygon shown in Figure 1, where $z, a, b, c, d, e$ are coarse points of control polygon. Let $\left\{p_{1}, p_{2}, \ldots, p_{n-1}\right\},\left\{p_{1}^{\prime}, p_{2}^{\prime}, \ldots, p_{n-1}^{\prime}\right\}$, and $\left\{p_{1}^{\prime \prime}, p_{2}^{\prime \prime}, \ldots, p_{n-1}^{\prime \prime}\right\}$ be the new inserted points (say DDpoints) by DD-scheme corresponding to the edges $\overrightarrow{a b}, \overrightarrow{b c}$ and $\overrightarrow{c d}$ respectively. Then second step is to modify all coarse points by using divided differences of coarse points and DD-points. Here we only discuss the rule to modify one point say $c$. The point $\mathrm{c}$ can be updated by following rule:

$$
c^{\prime}=\frac{p_{n-1}^{\prime}+p_{1}^{\prime \prime}-W_{c}}{2}
$$


where $W_{c}$ is the affine combination of second divided difference of coarse points and DD-points at point $c$ defined below:

$$
W_{c}=\mu\left\{\frac{d-2 c+b}{n^{2}}\right\}+(1-\mu)\left\{p_{1}^{\prime \prime}-2 c+p_{n-1}^{\prime}\right\}
$$

where $\mu \in[0,1]$ while

$$
p_{1}^{\prime \prime}=A_{1} b+A_{2} c+A_{3} d+A_{4} e, \quad p_{n-1}^{\prime}=A_{4} a+A_{3} b+A_{2} c+A_{1} d,
$$

where

$$
A_{1}=\frac{-(n-1)(2 n-1)}{6 n^{3}}, \quad A_{2}=\frac{\left(n^{2}-1\right)(2 n-1)}{2 n^{3}}, \quad A_{3}=\frac{(n+1)(2 n-1)}{2 n^{3}}, \quad A_{4}=\frac{-\left(n^{2}-1\right)}{6 n^{3}},
$$

and $n=2,3,4, \ldots$

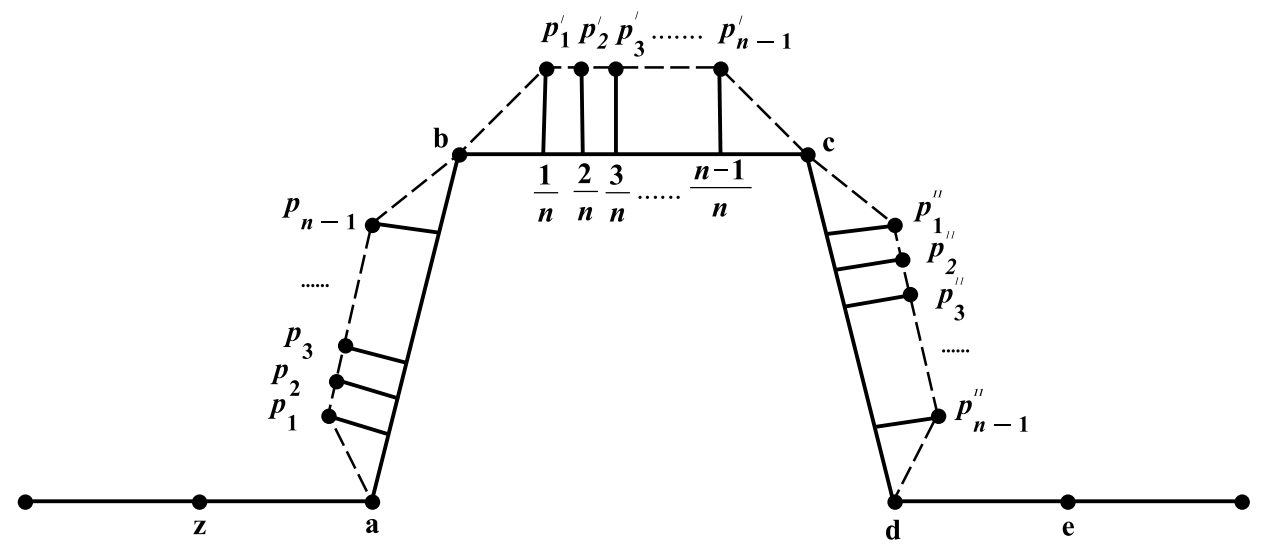

Figure 1: Labeling of a control polygon.

\subsection{Examples}

Here we see that 5 -point $n$-ary approximating schemes can be easily generated by above algorithm.

- By substituting $n=2$ in (2.1), we get the mask of 5-point binary approximating scheme of [1].

- If we substitute $n=3$ in (2.1), we get following 5-point ternary scheme

$$
\left\{\begin{array}{l}
f_{3 i}^{k+1}=-\frac{10}{162} f_{i-1}^{k}+\frac{120}{162} f_{i}^{k}+\frac{60}{162} f_{i+1}^{k}-\frac{8}{162} f_{i+2 \prime}^{k} \\
f_{3 i+1}^{k+1}=-\frac{8}{162} f_{i-1}^{k}+\frac{60}{16} f_{i}^{k}+\frac{120}{162} f_{i+1}^{k}-\frac{10}{162} f_{i+2}^{k} \\
f_{3 i+2}^{k+1}=-\frac{4 \mu}{162} f_{i-1}^{k}+\frac{16 \mu}{162} f_{i}^{k}+\frac{162-24 \mu}{162} f_{i+1}^{k}+\frac{16 \mu}{162} f_{i+2}^{k}-\frac{4 \mu}{162} f_{i+3}^{k} .
\end{array}\right.
$$

- For $n=4$ in (2.1), we get following 5-point quaternary scheme.

$$
\left\{\begin{array}{l}
f_{4 i}^{k+1}=-\frac{14}{256} f_{i-1}^{k}+\frac{210}{256} f_{i}^{k}+\frac{70}{256} f_{i+1}^{k}-\frac{10}{256} f_{i+2}^{k} \\
f_{4 i+1}^{k+1}=-\frac{16}{256} f_{i-1}^{k}+\frac{144}{256} f_{i}^{k}+\frac{144}{256} f_{i+1}^{k}-\frac{16}{256} f_{i+2}^{k} \\
f_{4 i+2}^{k+1}=-\frac{10}{256} f_{i-1}^{k}+\frac{70}{25} f_{i}^{k}+\frac{210}{256} f_{i+1}^{k}-\frac{14}{256} f_{i+2}^{k} \\
f_{4 i+3}^{k+1}=-\frac{5 \mu}{256} f_{i-1}^{k}+\frac{20 \mu}{256} f_{i}^{k}+\frac{256-30 \mu}{256} f_{i+1}^{k}+\frac{20 \mu}{256} f_{i+2}^{k}-\frac{5 \mu}{256} f_{i+3}^{k} .
\end{array}\right.
$$

- By substituting $\mu=0$ in (2.2) and (2.3), we get the mask of 4 -point ternary and quaternary interpolating scheme of [7]. 


\subsection{Smoothness analysis of proposed schemes}

We discuss the analysis of relaxed 5-point ternary and quaternary approximating subdivision schemes. We use the theory of generating function [9] to examine the convergence and smoothness of the scheme (2.2) and (2.3).

Theorem 2.1. The 5-point ternary approximating subdivision scheme (2.2) is $C^{3}$ for any $\mu \in(0.666,0.700)$.

Proof. The Laurent polynomial $\mathrm{a}(z)$ for the scheme (2.2) is

$$
\begin{aligned}
\mathrm{a}(z)= & \frac{1}{162}\left\{-4 \mu z^{0}-8 z^{1}-10 z^{2}+16 \mu z^{3}+60 z^{4}+120 z^{5}+(162-24 \mu) z^{6}\right. \\
& \left.+120 z^{7}+60 z^{8}+16 \mu z^{9}-10 z^{10}-8 z^{11}-4 \mu z^{12}\right\} .
\end{aligned}
$$

Now we consider

$$
c(z)=\left(\frac{3}{1+z+z^{2}}\right)^{4} a(z)=\frac{1}{2}\left(-4 \mu+(16 \mu-8) z+(22-24 \mu) z^{2}+(16 \mu-8) z^{3}-4 \mu z^{4}\right) .
$$

Note that

$$
\left\|\frac{1}{3} S_{c}\right\|_{\infty}=\frac{1}{3} \max \left\{\sum_{j \in \mathbb{Z}}\left|c_{3 j}\right|, \sum_{j \in \mathbb{Z}}\left|c_{3 j+1}\right|, \sum_{j \in \mathbb{Z}}\left|c_{3 j+2}\right|\right\} .
$$

For $\mu \in(0.666,0.700)$, we have

$$
\left\|\frac{1}{3} S_{c}\right\|_{\infty}=\frac{1}{3} \max \left\{\left|\frac{-4 \mu}{2}\right|+\left|\frac{16 \mu-8}{2}\right|,\left|\frac{-24 \mu+22}{2}\right|\right\}<1 .
$$

Hence $S_{c}$ is contractive. Therefore, by Corollary 4.17 of [9], the scheme (2.2) is $C^{3}$ for $\mu \in(0.666,0.700)$.

Theorem 2.2. The 5-point quaternary approximating subdivision scheme $(2.3)$ is $C^{2}$ for any $\mu$ in $(0.266,1)$.

Proof of the above theorem is similar to the proof of Theorem 2.1.

\section{Shape preserving properties}

In this section, we will discuss that what condition should be imposed on the initial points so that the limit curves generated by the subdivision schemes are monotonicity, convexity, and concavity preserving.

\subsection{Monotonicity preservation}

Definition 3.1 ([17]). A univariate data $\left(x_{i}, f_{i}\right), i=0,1,2, \ldots, n$ is monotonically increasing if $f_{i}<f_{i+1}$ $\forall i=0,1,2, \ldots, n$ and the derivative at the data points obey the condition $L_{i}=f_{i+1}-f_{i}>0 \forall i=$ $0,1,2, \ldots, n$.

Here, we examine monotonicity preservation of 5-point ternary approximating subdivision scheme (2.2) and 5-point quaternary approximating scheme (2.3).

Theorem 3.2. Let $\left\{f_{i}^{0}\right\}_{i \in \mathbb{Z}}$ be the sequence of initial points such that $f_{i}^{0}<f_{i+1}^{0}, i \in \mathbb{Z}$. Let

$$
L_{i}^{k}=f_{i+1}^{k}-f_{i}^{k}, \quad g_{i}^{k}=\frac{L_{i+1}^{k}}{L_{i}^{k}}, \quad G^{k}=\max _{i}\left\{g_{i}^{k}, \frac{1}{g_{i}^{k}}\right\}, \quad k \geqslant 0, k \in \mathbb{Z}, i \in \mathbb{Z} .
$$

Furthermore, let $0.3 \leqslant \mu \leqslant 1$ and $\xi=-\frac{1}{\mu}$,,$\in \mathbb{R}$. If $\frac{1}{\xi} \leqslant \mathrm{G}^{0} \leqslant \xi,\left\{\mathrm{f}_{i}^{\mathrm{k}}\right\}$ is defined by the subdivision scheme (2.2), then

$$
\mathrm{L}_{\mathrm{i}}^{\mathrm{k}}>0, \quad \frac{1}{\xi} \leqslant \mathrm{G}^{\mathrm{k}} \leqslant \xi, \quad k \geqslant 0, k \in \mathbb{Z}, i \in \mathbb{Z} .
$$


Proof. We use mathematical induction to prove (3.1). When $k=0, L_{i}^{0}=f_{i+1}^{0}-f_{i}^{0}>0, \frac{1}{\xi} \leqslant G^{0} \leqslant \xi$, then (3.1) is true. Suppose that (3.1) holds for $k$, i.e., $L_{i}^{k}=f_{i+1}^{k}-f_{i}^{k}>0, \frac{1}{\xi} \leqslant G^{k} \leqslant \xi$, since

$$
L_{3 i}^{k+1}=f_{3 i+1}^{k+1}-f_{3 i}^{k+1}=\frac{1}{81}\left\{-\left(f_{i}^{k}-f_{i-1}^{k}\right)+29\left(f_{i+1}^{k}-f_{i}^{k}\right)-\left(f_{i+2}^{k}-f_{i+1}^{k}\right)\right\} .
$$

This implies that

$$
\mathrm{L}_{3 i}^{\mathrm{k}+1}=\frac{1}{81}\left\{-\mathrm{L}_{\mathrm{i}-1}^{\mathrm{k}}+29 \mathrm{~L}_{\mathrm{i}}^{\mathrm{k}}-\mathrm{L}_{\mathrm{i}+1}^{\mathrm{k}}\right\}
$$

Similarly

$$
\begin{aligned}
& L_{3 i+1}^{k+1}=f_{3 i+2}^{k+1}-f_{3 i+1}^{k+1}=\frac{1}{81}\left\{(2 \mu-4) L_{i-1}^{k}+(26-6 \mu) L_{i}^{k}+(5+6 \mu) L_{i+1}^{k}-2 \mu L_{i+2}^{k}\right\}, \\
& L_{3 i+2}^{k+1}=f_{3 i+3}^{k+1}-f_{3 i+2}^{k+1}=\frac{1}{81}\left\{-2 \mu L_{i-1}^{k}+(5+6 \mu) L_{i}^{k}+(26-6 \mu) L_{i+1}^{k}+(2 \mu-4) L_{i+2}^{k}\right\} .
\end{aligned}
$$

Next we show that

$$
\mathrm{L}_{3 i}^{\mathrm{k}+1}>0, \quad \mathrm{~L}_{3 i+1}^{\mathrm{k}+1}>0, \text { and } \mathrm{L}_{3 i+2}^{\mathrm{k}+1}>0
$$

Now

$$
\mathrm{L}_{3 i}^{\mathrm{k}+1}=\frac{\mathrm{L}_{i}^{\mathrm{k}}}{81}\left\{-\frac{1}{\mathrm{~g}_{i-1}^{\mathrm{k}}}+29-\mathrm{g}_{i}^{\mathrm{k}}\right\}
$$

This implies

$$
\mathrm{L}_{3 i}^{\mathrm{k}+1} \geqslant \frac{\mathrm{L}_{i}^{\mathrm{k}}}{81}\{-2 \xi+29\}
$$

As we know that $L_{i}^{k}>0$ and

$$
\frac{1}{81}\{-2 \xi+29\}>0, \text { for } 0.3 \leqslant \mu \leqslant 1 \text { and } \xi=-\frac{1}{\mu} .
$$

This implies $L_{3 i}^{k+1}>0$. Similarly, we see that $L_{3 i+1}^{k+1}>0$, and $L_{3 i+2}^{k+1}>0$ for $0.3 \leqslant \mu \leqslant 1$ and $\xi=-\frac{1}{\mu}$.

Now we prove that $\frac{1}{\xi} \leqslant G^{k+1} \leqslant \xi$, we first show that $g_{3 i}^{k+1}-\xi \leqslant 0$.

$$
g_{3 i}^{k+1}=\frac{L_{3 i+1}^{k+1}}{L_{3 i}^{k+1}}=\frac{\frac{1}{81}\left\{(2 \mu-4) L_{i-1}^{k}+(26-6 \mu) L_{i}^{k}+(5+6 \mu) L_{i+1}^{k}-2 \mu L_{i+2}^{k}\right\}}{\frac{1}{81}\left\{-L_{i-1}^{k}+29 L_{i}^{k}-L_{i+1}^{k}\right\}} .
$$

This implies that

$$
\begin{aligned}
g_{3 i}^{k+1}-\xi= & \frac{1}{L_{i-1}^{k}\left\{-1+29 g_{i-1}^{k}-g_{i}^{k} g_{i-1}^{k}\right\}} L_{i}^{k}\left\{(2 \mu-4) \frac{1}{g_{i}^{k}}+(26-6 \mu)+(5+6 \mu) g_{i}^{k}\right. \\
& \left.-2 \mu g_{i+1}^{k} g_{i}^{k}+\xi \frac{1}{g_{i}^{k}}-29 \xi+\xi g_{i}^{k}\right\} .
\end{aligned}
$$

This further implies that

$$
g_{3 i}^{k+1}-\xi \leqslant \frac{L_{i}^{k}\left\{2 \xi^{2}+(8 \mu-28) \xi-8 \mu+26\right\}}{L_{i-1}^{k}\{29 \xi-2\}} .
$$


The denominator and numerator of right hand side of above expression are less than and greater than zero respectively for $0.3 \leqslant \mu \leqslant 1$ and $\xi=-\frac{1}{\mu}$. This implies that

$$
g_{3 i}^{k+1}-\xi \leqslant 0 .
$$

This further implies $g_{3 i}^{k+1} \leqslant \xi$. Now we show that $\frac{1}{g_{3 i}^{k+1}}-\xi \leqslant 0$.

$$
\frac{1}{g_{3 i}^{k+1}}-\xi \leqslant \frac{\frac{L_{i}^{k}}{81}\left\{2 \mu \xi^{3}+(6 \mu-26) \xi-2 \frac{1}{\xi}+28-8 \mu\right\}}{\left.\frac{L_{i+1}^{k}}{81}\left\{(2 \mu-4) \xi^{2}+(26-6 \mu) \xi-2 \mu \frac{1}{\xi}+6 \mu+5\right)\right\}} .
$$

The denominator and numerator of right hand side of above expression are less than and greater than zero respectively for $0.3 \leqslant \mu \leqslant 1$ and $\xi=-\frac{1}{\mu}$. This implies that

$$
\frac{1}{g_{3 i}^{k+1}}-\xi \leqslant 0 .
$$

This further implies $\frac{1}{g_{3 i}^{k+1}} \leqslant \xi$. In the same way, we see that $g_{3 i+1}^{k+1} \leqslant \xi, g_{3 i+2}^{k+1} \leqslant \xi, \frac{1}{g_{3 i+1}^{k+1}} \leqslant \xi$, and $\frac{1}{g_{3 i+2}^{k+1}} \leqslant \xi$. So $G^{k+1} \leqslant \xi$. Since $G^{k+1}=\max _{i}\left\{g_{i}^{k+1}, \frac{1}{g_{i}^{k+1}}\right\}$, it is obvious that $G^{k+1} \geqslant \frac{1}{\xi}$, which completes the proof.

Similarly, we can prove the following theorem.

Theorem 3.3. Let $\left\{f_{i}^{0}\right\}_{i \in \mathbb{Z}}$ be the sequence of initial points such that $f_{i}^{0}<f_{i+1}^{0}, i \in \mathbb{Z}$. Let

$$
L_{i}^{k}=f_{i+1}^{k}-f_{i}^{k}, \quad g_{i}^{k}=\frac{L_{i+1}^{k}}{L_{i}^{k}}, \quad G^{k}=\max _{i}\left\{g_{i}^{k}, \frac{1}{g_{i}^{k}}\right\}, k \geqslant 0, k \in \mathbb{Z}, i \in \mathbb{Z} .
$$

Furthermore, let $0.1 \leqslant \mu \leqslant 1$ and $\xi=-\frac{1}{\mu}, \xi \in \mathbb{R}$. If $\frac{1}{\xi} \leqslant \mathrm{G}^{0} \leqslant \xi,\left\{\mathrm{f}_{i}^{k}\right\}$ is defined by the subdivision scheme (2.3), then

$$
\mathrm{L}_{\mathrm{i}}^{\mathrm{k}}>0, \quad \frac{1}{\xi} \leqslant \mathrm{G}^{\mathrm{k}} \leqslant \xi, \quad k \geqslant 0, k \in \mathbb{Z}, i \in \mathbb{Z} .
$$

\subsection{Convexity preservation}

Definition 3.4 ([19]). Given a set of control points $\left\{p_{i}^{k}\right\}_{i \in \mathbb{Z}}, p_{i}^{k}=\left(x_{i}^{k}, f_{i}^{k}\right), f_{i}^{k}$ is strictly convex at a point $x_{i}^{k}$, if second order divided difference $d_{i}^{k}=f\left[x_{i-1}^{k}, x_{i}^{k}, x_{i+1}^{k}\right]>0$.

We prove the convexity preservation of subdivision schemes (2.2) and (2.3) with uniform initial control points. Given a set of initial control points $p_{i}^{0} \in \mathbb{Z}, p_{i}^{0}=\left(x_{i}^{0}, f_{i}^{0}\right)$ which are strictly convex, where $x_{i}^{0} \in \mathbb{Z}$ are equidistant points. To simplify, we use $\triangle x_{i}^{0}=x_{i+1}^{0}-x_{i}^{0}=1$. By the subdivision scheme (2.2), we have $\Delta x_{i}^{k+1}=x_{i+1}^{k+1}-x_{i}^{k+1}=\frac{1}{3} \Delta x_{i}^{k}=\frac{1}{3^{k+1}}$. Denote $d_{i}^{k}=f\left[x_{i-1}^{k}, x_{i}^{k}, x_{i+1}^{k}\right]=3^{2 k} 2^{-1}\left(f_{i-1}^{k}-2 f_{i}^{k}+f_{i+1}^{k}\right)$ as the second order divided differences. By the subdivision scheme (2.3), we have $\triangle x_{i}^{k+1}=x_{i+1}^{k+1}-x_{i}^{k+1}=$ $\frac{1}{4} \triangle x_{i}^{k}=\frac{1}{4^{k+1}}$. Denote $d_{i}^{k}=f\left[x_{i-1}^{k}, x_{i}^{k}, x_{i+1}^{k}\right]=4^{2 k} 2^{-1}\left(f_{i-1}^{k}-2 f_{i}^{k}+f_{i+1}^{k}\right)$ as the second order divided differences. In the following, we will prove $d_{i}^{k}>0, \forall k \geqslant 0, k \in \mathbb{Z}, i \in \mathbb{Z}$.

Theorem 3.5. Suppose that the initial control points $\left\{p_{i}^{0}\right\}_{i \in \mathbb{Z}},\left\{p_{i}^{0}\right\}=\left(x_{i}^{0}, f_{i}^{0}\right)$ are strictly convex, i.e., $d_{i}^{0}>0$, for all $i \in \mathbb{Z}$. Let

$$
\begin{aligned}
& d_{i}^{k}=3^{2 k}(2)^{-1}\left(f_{i-1}^{k}-2 f_{i}^{k}+f_{i+1}^{k}\right), \quad y_{i}^{k}=\frac{d_{i+1}^{k}}{d_{i}^{k}}, \\
& Y^{k}=\max _{i}\left\{y_{i}^{k}, \frac{1}{y_{i}^{k}}\right\}, \quad \forall k \geqslant 0, \quad k \in \mathbb{Z}, \quad i \in \mathbb{Z} .
\end{aligned}
$$


Furthermore, let $0.3 \leqslant \mu \leqslant 0.9$ and $\delta=\frac{1}{\mu}, \delta \in \mathbb{R}$. Then for $\frac{1}{\delta} \leqslant Y^{0} \leqslant \delta$,

$$
\mathrm{d}_{\mathrm{i}}^{\mathrm{k}}>0, \quad \frac{1}{\delta} \leqslant \mathrm{Y}^{\mathrm{k}} \leqslant \delta, \forall \mathrm{k} \geqslant 0, k \in \mathbb{Z}, i \in \mathbb{Z} .
$$

That is, the limit function generated by the subdivision scheme (2.2) is strictly convex.

Proof. We use mathematical induction to prove (3.2). When $k=0, d_{i}^{0}>0, \frac{1}{\delta} \leqslant \gamma^{0} \leqslant \delta$, then (3.2) is true. Suppose that (3.2) holds for $k$, i.e., $d_{i}^{k}>0, \frac{1}{\delta} \leqslant Y^{k} \leqslant \delta$, since

$$
d_{3 i}^{k+1}=3^{2 k}(2)^{-1}\left(f_{3 i-1}^{k+1}-2 f_{3 i}^{k+1}+f_{3 i+1}^{k+1}\right) .
$$

This implies that

$$
d_{3 i}^{k+1}=-\frac{2 \mu}{9} d_{i-1}^{k}+\left(\frac{2}{3}+\frac{4 \mu}{9}\right) d_{i}^{k}+\left(\frac{1}{3}-\frac{2 \mu}{9}\right) d_{i+1}^{k} .
$$

Similarly

$$
\begin{aligned}
& d_{3 i+1}^{k+1}=\left(-\frac{4}{9}+\frac{4 \mu}{9}\right) d_{i-1}^{k}+\left(\frac{17}{9}-\frac{8 \mu}{9}\right) d_{i}^{k}+\left(-\frac{4}{9}+\frac{4 \mu}{9}\right) d_{i+1}^{k}, \\
& d_{3 i+2}^{k+1}=\left(\frac{1}{3}-\frac{2 \mu}{9}\right) d_{i-1}^{k}+\left(\frac{2}{3}+\frac{4 \mu}{9}\right) d_{i}^{k}-\frac{2 \mu}{9} d_{i+1}^{k} .
\end{aligned}
$$

Next we show that

$$
d_{3 i}^{k+1}>0, d_{3 i+1}^{k+1}>0, \text { and } d_{3 i+2}^{k+1}>0
$$

Since

$$
d_{3 i}^{k+1}=d_{i}^{k}\left\{-\frac{2 \mu}{9} \frac{1}{y_{i-1}^{k}}+\left(\frac{2}{3}+\frac{4 \mu}{9}\right)+\left(\frac{1}{3}-\frac{2 \mu}{9}\right) y_{i}^{k}\right\}
$$

Then

$$
d_{3 i}^{k+1} \geqslant d_{i}^{k}\left\{-\frac{2 \mu}{9} \delta+\left(\frac{2}{3}+\frac{4 \mu}{9}\right)+\left(\frac{1}{3}-\frac{2 \mu}{9}\right) \frac{1}{\delta}\right\} .
$$

As we know that $d_{i}^{k}>0$ and

$$
\left\{-\frac{2 \mu}{9} \delta+\left(\frac{2}{3}+\frac{4 \mu}{9}\right)+\left(\frac{1}{3}-\frac{2 \mu}{9}\right) \frac{1}{\delta}\right\}>0, \text { for } 0.3 \leqslant \mu \leqslant 0.9 \text { and } \delta=\frac{1}{\mu} .
$$

This implies that $d_{3 i}^{k+1}>0$. Similarly, we can prove that $d_{3 i+1}^{k+1}>0$ and $d_{3 i+2}^{k+1}>0$ for $0.3 \leqslant \mu \leqslant 0.9$ and $\delta=\frac{1}{\mu}$.

Now we prove that $\frac{1}{\delta} \leqslant \gamma^{k+1} \leqslant \delta$, we first show that $y_{3 i}^{k+1}-\delta \leqslant 0$.

$$
y_{3 i}^{k+1}=\frac{d_{3 i+1}^{k+1}}{d_{3 i}^{k+1}}=\frac{\left(-\frac{4}{9}+\frac{4 \mu}{9}\right) d_{i-1}^{k}+\left(\frac{17}{9}-\frac{8 \mu}{9}\right) d_{i}^{k}+\left(-\frac{4}{9}+\frac{4 \mu}{9}\right) d_{i+1}^{k}}{-\frac{2 \mu}{9} d_{i-1}^{k}+\left(\frac{2}{3}+\frac{4 \mu}{9}\right) d_{i}^{k}+\left(\frac{1}{3}-\frac{2 \mu}{9}\right) d_{i+1}^{k}} .
$$

This implies that

$$
y_{3 i}^{k+1}-\delta \leqslant \frac{d_{i}^{k}\left\{\frac{2 \mu}{9} \delta^{2}+\left(\frac{4 \mu}{9}-\frac{14}{9}\right) \delta-\frac{6 \mu}{9}+\frac{14}{9}\right\}}{d_{i-1}^{k}\left\{\left(\frac{1}{3}-\frac{2 \mu}{9}\right) \delta^{2}+\left(\frac{2}{3}+\frac{4 \mu}{9}\right) \delta-\frac{2 \mu}{9}\right\}} .
$$


Since denominator and numerator of right hand side of above expression are greater than and less than zero respectively for $0.3 \leqslant \mu \leqslant 0.9$ and $\delta=\frac{1}{\mu}$, then

$$
y_{3 i}^{k+1}-\delta \leqslant 0 .
$$

This implies that $y_{3 i}^{k+1} \leqslant \delta$. Now we show that $\frac{1}{y_{3 i}^{k+1}}-\delta \leqslant 0$.

$$
\frac{1}{y_{3 i}^{k+1}}=\frac{d_{3 i}^{k+1}}{d_{3 i+1}^{k+1}}=\frac{-\frac{2 \mu}{9} d_{i-1}^{k}+\left(\frac{2}{3}+\frac{4 \mu}{9}\right) d_{i}^{k}+\left(\frac{1}{3}-\frac{2 \mu}{9}\right) d_{i+1}^{k}}{\left(-\frac{4}{9}+\frac{4 \mu}{9}\right) d_{i-1}^{k}+\left(\frac{17}{9}-\frac{8 \mu}{9}\right) d_{i}^{k}+\left(-\frac{4}{9}+\frac{4 \mu}{9}\right) d_{i+1}^{k}} .
$$

This implies that

$$
\frac{1}{y_{3 i}^{k+1}}-\delta \leqslant \frac{d_{i}^{k}\left\{\left(-\frac{14}{9}+\frac{2 \mu}{3}\right) \delta-\frac{2 \mu}{9} \frac{1}{\delta}-\frac{4 \mu}{9}+\frac{14}{9}\right\}}{d_{i-1}^{k}\left\{\left(-\frac{4}{9}+\frac{4 \mu}{9}\right)+\left(\frac{17}{9}-\frac{8 \mu}{9}\right) \delta+\left(-\frac{4}{9}+\frac{4 \mu}{9}\right) \delta^{2}\right\}} .
$$

Again denominator and numerator of right hand side of above expression are greater than and less than zero respectively for $0.3 \leqslant \mu \leqslant 0.9$ and $\delta=\frac{1}{\mu}$ so

$$
\frac{1}{y_{3 i}^{k+1}}-\delta \leqslant 0
$$

This implies $\frac{1}{y_{3 i}^{k+1}} \leqslant \delta$. In the same way, we see that $y_{3 i+1}^{k+1} \leqslant \delta, y_{3 i+2}^{k+1} \leqslant \delta, \frac{1}{y_{3 i+1}^{k+1}} \leqslant \delta$, and $\frac{1}{y_{3 i+2}^{k+1}} \leqslant \delta$. So $Y^{k+1} \leqslant \delta$. Since $Y^{k+1}=\max _{i}\left\{y_{i}^{k+1}, \frac{1}{y_{i}^{k+1}}\right\}$, it is obvious that $Y^{k+1} \geqslant \frac{1}{\delta}$, which completes the proof.

Similarly, we can prove the following theorem.

Theorem 3.6. Suppose that the initial control points $\left\{p_{i}^{0}\right\}_{i \in \mathbb{Z}},\left\{p_{i}^{0}\right\}=\left(x_{i}^{0}, f_{i}^{0}\right)$ are strictly convex, i.e., $d_{i}^{0}>0$, for all $i \in \mathbb{Z}$. Let

$$
\begin{aligned}
& d_{i}^{k}=4^{2 k}(2)^{-1}\left(f_{i-1}^{k}-2 f_{i}^{k}+f_{i+1}^{k}\right), \quad y_{i}^{k}=\frac{d_{i+1}^{k}}{d_{i}^{k}}, \\
& Y^{k}=\max _{i}\left\{y_{i}^{k}, \frac{1}{y_{i}^{k}}\right\}, \quad \forall k \geqslant 0, \quad k \in \mathbb{Z}, \quad i \in \mathbb{Z} .
\end{aligned}
$$

Furthermore, let $0.1 \leqslant \mu \leqslant 0.9$ and $\delta=-\frac{1+\mu}{2 \mu}, \delta \in \mathbb{R}$. Then for $\frac{1}{\delta} \leqslant \gamma^{0} \leqslant \delta$,

$$
\mathrm{d}_{i}^{\mathrm{k}}>0, \quad \frac{1}{\delta} \leqslant \mathrm{Y}^{\mathrm{k}} \leqslant \delta, \forall \mathrm{k} \geqslant 0, k \in \mathbb{Z}, i \in \mathbb{Z} .
$$

That is, the limit function generated by the subdivision scheme (2.3) is strictly convex.

3.3. Concavity preservation

Definition 3.7 ([19]). Given a set of control points $\left\{p_{i}^{k}\right\}_{i \in \mathbb{Z}}, p_{i}^{k}=\left(x_{i}^{k}, f_{i}^{k}\right), f_{i}^{k}$ is strictly concave at a point $x_{i}^{k}$, if second order divided difference $d_{i}^{k}=f\left[x_{i-1}^{k}, x_{i}^{k}, x_{i+1}^{k}\right]<0$. points.

We prove the concavity preservation of subdivision schemes (2.2) and (2.3) with uniform initial control

Theorem 3.8. Suppose that the initial control points $\left\{p_{i}^{0}\right\}_{i \in \mathbb{Z}},\left\{p_{i}^{0}\right\}=\left(\chi_{i}^{0}, f_{i}^{0}\right)$ are strictly concave, i.e., $d_{i}^{0}<0$ for all $i \in \mathbb{Z}$. Let

$$
\begin{aligned}
d_{i}^{k} & =3^{2 k}(2)^{-1}\left(f_{i-1}^{k}-2 f_{i}^{k}+f_{i+1}^{k}\right), \quad q_{i}^{k}=\frac{d_{i+1}^{k}}{d_{i}^{k}}, \\
Q^{k} & =\max _{i}\left\{q_{i}^{k}, \frac{1}{q_{i}^{k}}\right\}, \quad \forall k \geqslant 0, \quad k \in \mathbb{Z}, \quad i \in \mathbb{Z} .
\end{aligned}
$$

Furthermore, let $0.3 \leqslant \mu \leqslant 0.9$ and $\beta=\frac{1}{\mu}, \beta \in \mathbb{R}$. Then for $\frac{1}{\beta} \leqslant Q^{0} \leqslant \beta$, 


$$
d_{i}^{k}<0, \quad \frac{1}{\beta} \leqslant Q^{k} \leqslant \beta, \forall k \geqslant 0, k \in \mathbb{Z}, i \in \mathbb{Z}
$$

Namely, the limit function generated by the subdivision scheme (2.2) is strictly concave.

Proof. We use mathematical induction to prove (3.4). When $k=0, d_{i}^{0}<0$ and $\frac{1}{\beta} \leqslant Q^{0} \leqslant \beta$, then (3.4) is true. Suppose that (3.4) holds for $k$, i.e., $d_{i}^{k}<0, \frac{1}{\beta} \leqslant Q^{k} \leqslant \beta$.

Next we show that

$$
d_{3 i}^{k+1}<0, \quad d_{3 i+1}^{k+1}<0, \text { and } d_{3 i+2}^{k+1}<0
$$

From (3.3), we have

$$
d_{3 i}^{k+1} \leqslant d_{i}^{k}\left\{-\frac{2 \mu}{9} \frac{1}{\beta}+\left(\frac{2}{3}+\frac{4 \mu}{9}\right)+\left(\frac{1}{3}-\frac{2 \mu}{9}\right) \beta\right\}
$$

As we know that $d_{i}^{k}<0$ and

$$
\left\{-\frac{2 \mu}{9} \frac{1}{\beta}+\left(\frac{2}{3}+\frac{4 \mu}{9}\right)+\left(\frac{1}{3}-\frac{2 \mu}{9}\right) \beta\right\}>0, \text { for } 0.3 \leqslant \mu \leqslant 0.9 \text { and } \beta=\frac{1}{\mu} \text {. }
$$

This implies that $d_{3 i}^{k+1}<0$. Similarly, we see that $d_{3 i+1}^{k+1}<0$ and $d_{3 i+2}^{k+1}<0$ for $0.3 \leqslant \mu \leqslant 0.9$ and $\beta=\frac{1}{\mu}$.

Now we prove that $\frac{1}{\beta} \leqslant Q^{k+1} \leqslant \beta$, first we show that $q_{3 i}^{k+1}-\beta \leqslant 0$. Since

$$
q_{3 i}^{k+1}-\beta \leqslant \frac{d_{i}^{k}\left\{\frac{2 \mu}{9} \beta^{2}+\left(\frac{4 \mu}{9}-\frac{14}{9}\right) \beta-\frac{6 \mu}{9}+\frac{14}{9}\right\}}{d_{i-1}^{k}\left\{\left(\frac{1}{3}-\frac{2 \mu}{9}\right) \beta^{2}+\left(\frac{2}{3}+\frac{4 \mu}{9}\right) \beta-\frac{2 \mu}{9}\right\}} .
$$

The denominator and numerator of right hand side of above expression are less than and greate than zero respectively for $0.3 \leqslant \mu \leqslant 0.9$ and $\beta=\frac{1}{\mu}$. This implies that

$$
q_{3 i}^{k+1}-\beta \leqslant 0 .
$$

This further implies $q_{3 i}^{k+1} \leqslant \beta$. Now we show that $\frac{1}{q_{3 i}^{k+1}}-\beta \leqslant 0$.

$$
\frac{1}{\mathrm{q}_{3 i}^{\mathrm{k}+1}}-\beta \leqslant \frac{\mathrm{d}_{\mathrm{i}}^{\mathrm{k}}\left\{\left(-\frac{14}{9}+\frac{2 \mu}{3}\right) \beta-\frac{2 \mu}{9} \frac{1}{\beta}-\frac{4 \mu}{9}+\frac{14}{9}\right\}}{\mathrm{d}_{\mathrm{i}-1}^{\mathrm{k}}\left\{\left(-\frac{4}{9}+\frac{4 \mu}{9}\right) \beta^{2}+\left(\frac{17}{9}-\frac{8 \mu}{9}\right) \beta+\left(-\frac{4}{9}+\frac{4 \mu}{9}\right)\right\}} .
$$

The denominator and numerator of right hand side of above expression are less than and greater than zero respectively for $0.3 \leqslant \mu \leqslant 0.9$ and $\beta=\frac{1}{\mu}$. This implies that

$$
\frac{1}{q_{3 i}^{k+1}}-\beta \leqslant 0
$$

This further implies that $\frac{1}{q_{3 i}^{k+1}} \leqslant \beta$. In the same way, we can get $q_{3 i+1}^{k+1} \leqslant \beta, q_{3 i+2}^{k+1} \leqslant \beta, \frac{1}{q_{3 i+1}^{k+1}} \leqslant \beta$, and $\frac{1}{\mathrm{q}_{3 i+2}^{k+1}} \leqslant \beta$. So $\mathrm{Q}^{\mathrm{k}+1} \leqslant \beta$. Since $\mathrm{Q}^{\mathrm{k}+1}=\max _{i}\left\{\mathrm{q}_{i}^{k+1}, \frac{1}{\mathrm{q}_{i}^{k+1}}\right\}$, it is obvious that $\mathrm{Q}^{k+1} \geqslant \frac{1}{\beta}$, which completes the proof.

Similarly, we have following theorem. 
Theorem 3.9. Suppose that the initial control points $\left\{p_{i}^{0}\right\}_{i \in \mathbb{Z}},\left\{p_{i}^{0}\right\}=\left(\chi_{i}^{0}, f_{i}^{0}\right)$ are strictly concave, i.e., $d_{i}^{0}<0$ for all $i \in \mathbb{Z}$. Let

$$
\begin{aligned}
& d_{i}^{k}=4^{2 k}(2)^{-1}\left(f_{i-1}^{k}-2 f_{i}^{k}+f_{i+1}^{k}\right), \quad q_{i}^{k}=\frac{d_{i+1}^{k}}{d_{i}^{k}}, \\
& Q^{k}=\max _{i}\left\{q_{i}^{k}, \frac{1}{q_{i}^{k}}\right\}, \quad \forall k \geqslant 0, \quad k \in \mathbb{Z}, \quad i \in \mathbb{Z} .
\end{aligned}
$$

Furthermore, let $0.1 \leqslant \mu \leqslant 0.9$ and $\beta=-\frac{2 \mu}{1+\mu}, \beta \in \mathbb{R}$. Then for $\frac{1}{\beta} \leqslant Q^{0} \leqslant \beta$,

$$
d_{i}^{k}<0, \quad \frac{1}{\beta} \leqslant Q^{k} \leqslant \beta, \forall k \geqslant 0, k \in \mathbb{Z}, i \in \mathbb{Z}
$$

Namely, the limit function generated by the subdivision scheme (2.3) is strictly concave.

Table 1: Monotone data set.
\begin{tabular}{|llllll}
$\mathrm{x}$ & 5 & 7 & 11 & 12 & 16 \\
$\mathrm{y}$ & 10 & 11 & 15 & 50 & 85 \\
\hline
\end{tabular}

Table 2: Convex data set.

\begin{tabular}{llllllll}
\hline $\mathrm{x}$ & 0 & 1 & 2 & 3 & 4 & 5 & 6 \\
$\mathrm{y}$ & 9 & 5 & 3 & 2.40 & 2.20 & 2.15 & 2.10 \\
\hline
\end{tabular}

\begin{tabular}{llllllllll}
\multicolumn{10}{c}{ Table 3: Concave data set. } \\
\hline $\mathrm{x}$ & 1 & 2 & 3 & 4 & 5 & 6 & 7 & 8 & 9 \\
$\mathrm{y}$ & 0 & 0.301 & 0.477 & 0.602 & 0.698 & 0.778 & 0.845 & 0.903 & 0.954 \\
\hline
\end{tabular}

\subsection{Demonstration}

In this section, a numerical demonstration of monotonicity, convexity, and concavity preserving schemes given in previous section is presented.

Example 3.10. Figures 2 and 3 are produced by using monotone data set presented in Table 1 borrowed by [17]. In Figure 2 (a), curve is generated by using cubic Hermite spline which looses the monotone shape of the data, Figures 2 (b)-(e) are monotone curves obtained by rational cubic function [16], [31], schemes (2.2) and (2.3) at $\mu=0.5$, respectively. It must be noted that rational cubic function tightly fit the data while schemes (2.2) and (2.3) have relaxed data fitting. Scheme by [31] also has too relaxed data fitting. The monotonicity preservation of proposed schemes (2.2) and (2.3) shown in Figures 2 (d)-(e) give the better results as compare to the existing schemes cubic Hermite spline, [16] and [31] shown in Figures 2 (a)-(c).

Figures 3 (a-c) are generated by [16], schemes (2.2) and (2.3) at different values of parameter. From these figures it is observed that parameter in rational function of [16] effect the fitting over some part of the domain while fitting remain tight over other part of the domain. Schemes (2.2) and (2.3) give loose/tight fitting at different values of parameter. These schemes also preserve the shape of data.

Example 3.11. Convex data set is given in Table 2 [27] and Figure 4 is produced by using this data. Figures 4 (a)-(f) are convex curves obtained by $[3,8,14,31]$, schemes (2.2) and (2.3) for $\mu=0.5$, respectively. In 
Figure 4 (a), Curve is far away from initial and final points due to high shrinkage effect of the scheme. Curve is nicely approximately fitted over the data in Figure 4 (b). It is to be noted that in Figure 4 (c), curve exhibits slight fluctuation over the part of domain, i.e., over (2 3). Fluctuation area is shown by a circle. In Figures 4 (d)-(e), curves have interpolatory effect and smoothly pass through the control points. The convexity preservation of proposed schemes (2.2) and (2.3) shown in Figures 4 (e)-(f) give the better results as compare to the existing schemes [14, 31], and [3] shown in Figures 4 (a)-(c).

Example 3.12. Table 3 shows the concave data set by using the function $y=\log x$ and Figure 5 is produced by using this data. Concave curves shown in Figures 5 (a)-(b) are produced by schemes (2.2) and (2.3) at $\mu=0.5$, respectively.

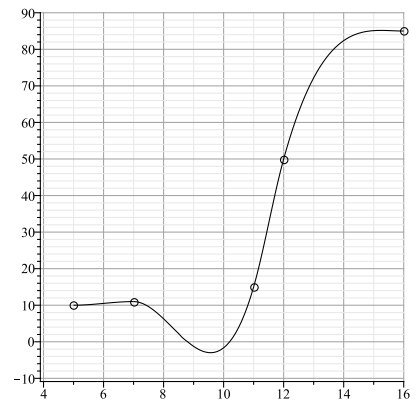

(a)

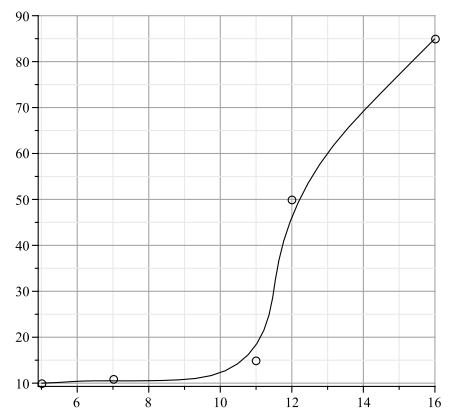

(d)

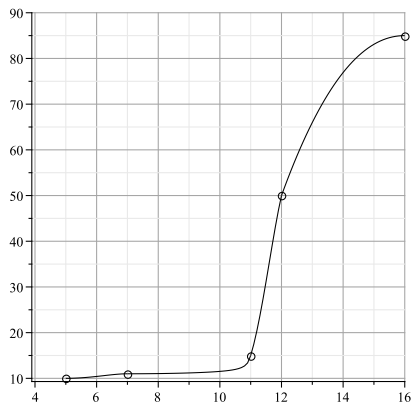

(b)

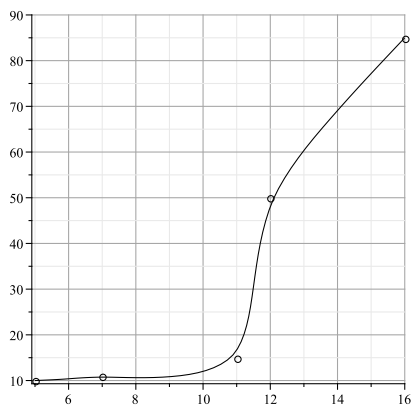

(e)

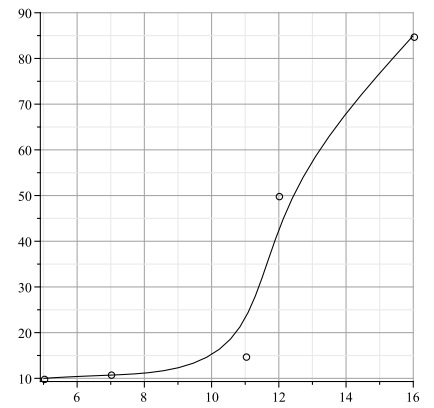

(c)

Figure 2: The curves (a), (b), (c), (d), and (e) are generated by cubic Hermite spline, [16, 31], schemes (2.2), and (2.3) by using monotone data set.

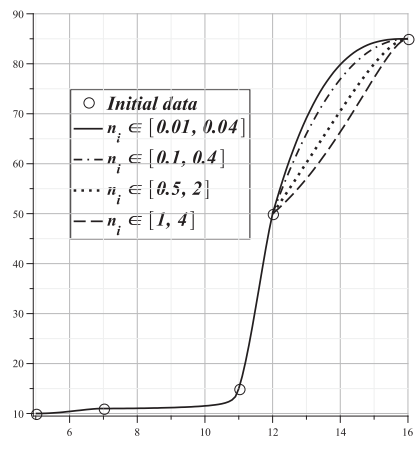

(a)

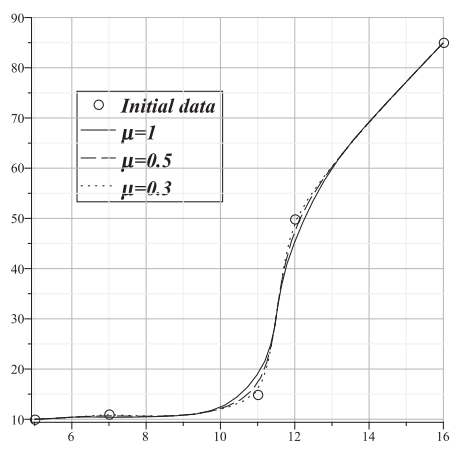

(b)

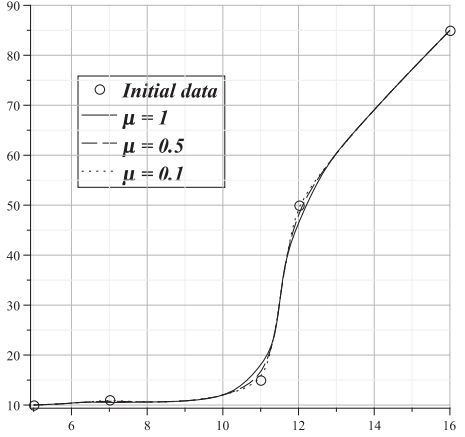

(c)

Figure 3: The curves (a), (b), and (c) are generated by rational cubic function [16], schemes (2.2), and (2.3), respectively by using monotone data set. 


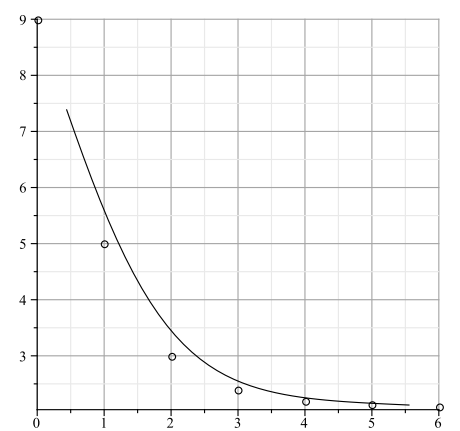

(a)

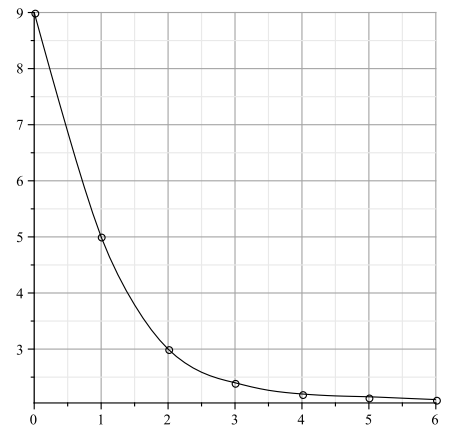

(d)

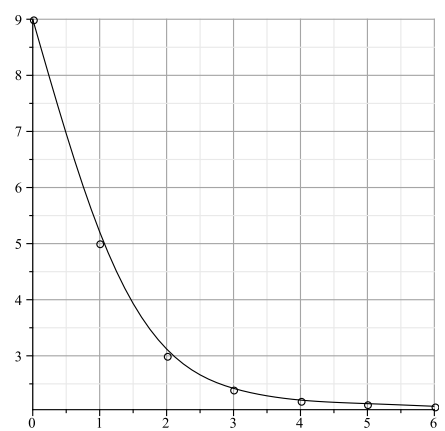

(b)

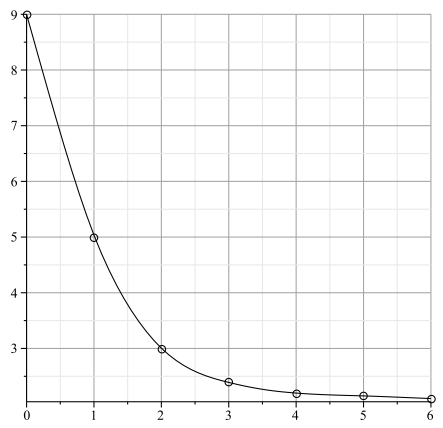

(e)

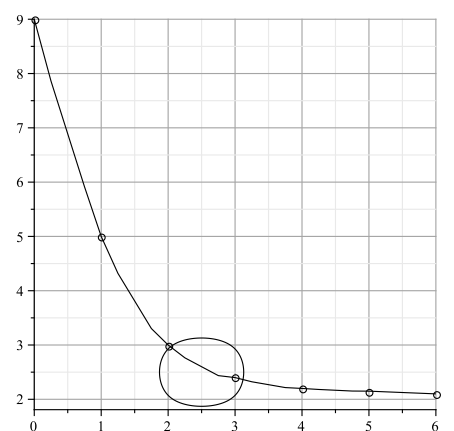

(c)

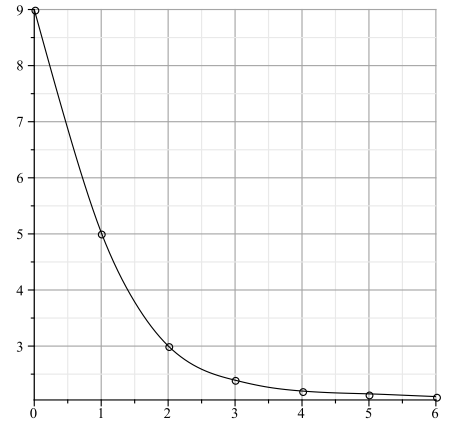

(f)

Figure 4: The convex curves (a), (b), (c), (d), and (e) are generated by [3, 8, 14, 31], schemes (2.2), and (2.3), respectively by using convex data set.

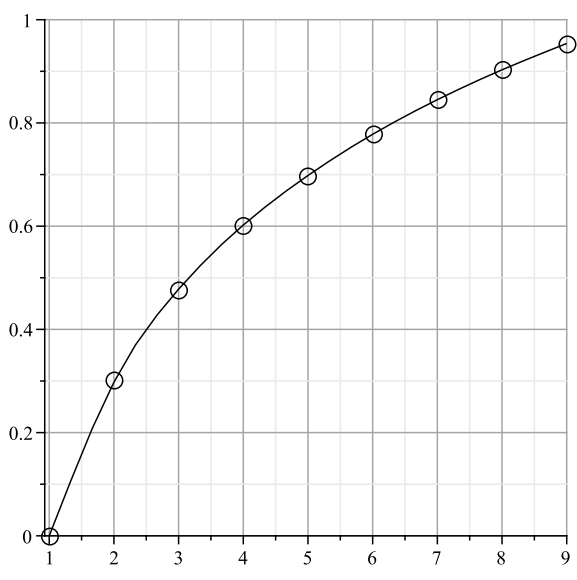

(a)

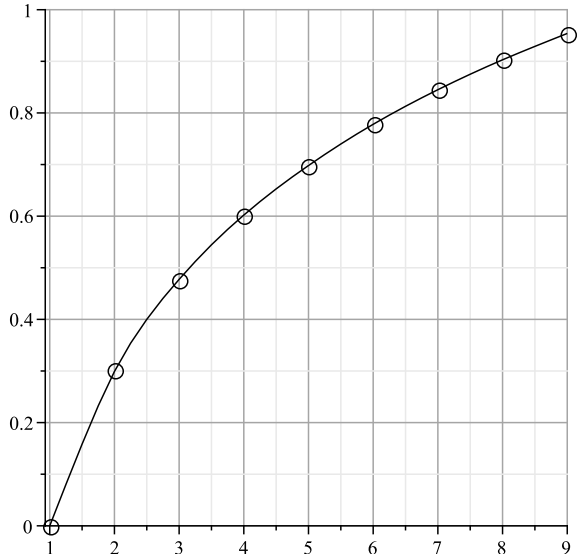

(b)

Figure 5: The concave curves (a) and (b) are generated by scheme (2.2) and (2.3), respectively by using concave data set.

\section{Traditional properties of schemes}

In this section, we discuss some significant properties like Hölder exponent, polynomial generation, polynomial reproduction, approximation order, and support width of basic limit function of the schemes (2.2) and (2.3) by using techniques developed in $[5,9,21,26]$.

- The Hölder exponents of schemes (2.2) and (2.3) are 3.052 and 2.888, respectively. Figure 6 (a) shows a graph of the Hölder exponent against $\mu \in(0.666,0.7)$. Notice that the highest smoothness of the 5-point ternary scheme (2.2) is achieved at $\mu=0.683$ and its Hölder exponent is $H=3.052$. Figure 
6 (b) shows a graph of the Hölder exponent against $\mu \in(0.266,1)$. It also shuold be noted that the highest smoothness of the 5-point quaternary scheme (2.3) is achieved at $\mu=0.633$ and its Hölder exponent is $\mathrm{H}=2.888$.

- The basic function defined by relaxed 5-point $n$-ary approximating scheme has support width $\mathrm{S}=$ $\frac{4 n}{n-1}$, which implies that it vanishes outside the interval $\left[-\frac{4 n}{2(n-1)}, \frac{4 n}{2(n-1)}\right]$.

- The degree of polynomial generation of schemes (2.2) and (2.3) is 3.

- Schemes (2.2) and (2.3) reproduce polynomials of degree 3.

- Subdivision schemes (2.2) and (2.3) have approximation order 4.

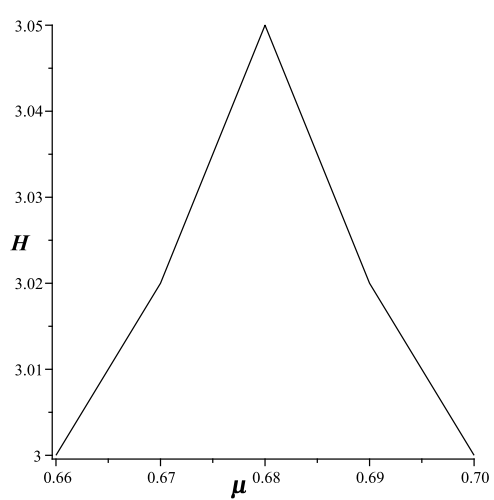

(a)

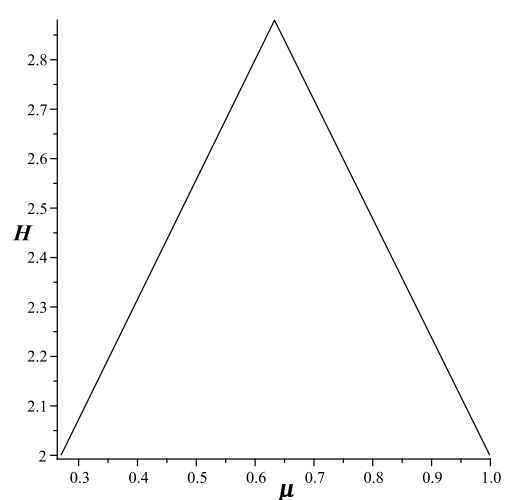

(b)

Figure 6: (a) Graph of the Hölder exponent against $\mu$ for the scheme (2.2). (b) Graph of the Hölder exponent against $\mu$ for the scheme (2.3).

In following theorem, we show that the basic limit function of 5-point $n$-ary scheme is symmetric.

Theorem 4.1. The basic limit function of 5-point $\mathrm{n}$-ary scheme is symmetric about $Y$-axis.

Proof. Let $F$ denotes the basic limit function and define $R_{k}:=\left\{\frac{i}{n^{k}} \mid i \in \mathbb{Z}\right\}$ such that the restriction of the basic limit function $F$ to $R_{k}$ satisfies $F\left(\frac{i}{n^{k}}\right)=f_{i}^{k}$ for all $i \in \mathbb{Z}$. The symmetry of basic function is proved using mathematical induction on $\mathrm{k}$.

First of all we note that $F(i)=f_{i}^{0}=f_{-i}^{0}=F(-i)$ for all $i \in \mathbb{Z}$ and thus $F\left(\frac{i}{n^{k}}\right)=F\left(-\frac{i}{n^{k}}\right)=f_{-i}^{0}$ for all $i \in \mathbb{Z}, \quad k=0$.

Now we assume that $F\left(\frac{i}{n^{k}}\right)=F\left(-\frac{i}{n^{k}}\right)$ for all $i \in \mathbb{Z}$, then it follows that $f_{i}^{k}=F\left(\frac{i}{n^{k}}\right)=F\left(-\frac{i}{n^{k}}\right)=f_{-i}^{k}$ for all $i \in \mathbb{Z}$. For $\alpha=0,1, \ldots, n-2$, we have

$$
f_{n i+\alpha}^{k+1}=A_{\alpha}^{n} f_{i-1}^{k}+B_{\alpha}^{n} f_{i}^{k}+C_{\alpha}^{n} f_{i+1}^{k}+D_{\alpha}^{n} f_{i+2}^{k}
$$

for $\alpha=n-1$

$$
f_{n i+n-1}^{k+1}=E_{1}^{n} f_{i-1}^{k}+E_{2}^{n} f_{i}^{k}+E_{3}^{n} f_{i+1}^{k}+E_{2}^{n} f_{i+2}^{k}+E_{1}^{n} f_{i+3}^{k} .
$$

Let $A_{\alpha}^{n}, B_{\alpha}^{n}, C_{\alpha}^{n}, D_{\alpha}^{n}, E_{1}^{n}, E_{2}^{n}$, and $E_{3}^{n}$ are the coefficients of the 5-point $n$-ary approximating scheme. Therefore

$$
F\left(\frac{n i}{n^{k+1}}\right)=f_{n i}^{k+1}=A_{0}^{n} f_{i-1}^{k}+B_{0}^{n} f_{i}^{k}+C_{0}^{n} f_{i+1}^{k}+D_{0}^{n} f_{i+2}^{k}
$$


This implies that

$$
F\left(\frac{n i}{n^{k+1}}\right)=A_{0}^{n} F\left(\frac{i-1}{n^{k}}\right)+B_{0}^{n} F\left(\frac{i}{n^{k}}\right)+C_{0}^{n} F\left(\frac{i+1}{n^{k}}\right)+D_{0}^{n} F\left(\frac{i+2}{n^{k}}\right)
$$

So we have

$$
F\left(\frac{n i}{n^{k+1}}\right)=A_{0}^{n} F\left(\frac{-(i-1)}{n^{k}}\right)+B_{0}^{n} F\left(\frac{-i}{n^{k}}\right)+C_{0}^{n} F\left(\frac{-(i+1)}{n^{k}}\right)+D_{0}^{n} F\left(\frac{-(i+2)}{n^{k}}\right) .
$$

Thus we have

$$
F\left(\frac{n i}{n^{k+1}}\right)=A_{0}^{n} f_{-(i-1)}^{k}+B_{0}^{n} f_{-i}^{k}+C_{0}^{n} f_{-(i+1)}^{k}+D_{0}^{n} f_{-(i+2)}^{k}=f_{-n i}^{k+1}=F\left(-\frac{n i}{n^{k+1}}\right) .
$$

Similarly, we can easily show that for $\alpha=1,2, \ldots, n-2$,

$$
F\left(\frac{n i+\alpha}{n^{k+1}}\right)=F\left(-\frac{n i+\alpha}{n^{k+1}}\right) .
$$

For $\alpha=\mathrm{n}-1$, we have

$$
F\left(\frac{n i+n-1}{n^{k+1}}\right)=f_{n i+n-1}^{k+1}=E_{1}^{n} f_{i-1}^{k}+E_{2}^{n} f_{i}^{k}+E_{3}^{n} f_{i+1}^{k}+E_{2}^{n} f_{i+2}^{k}+E_{1}^{n} f_{i+3}^{k} .
$$

This implies that

$$
F\left(\frac{n i+n-1}{n^{k+1}}\right)=E_{1}^{n} F\left(\frac{i-1}{n^{k}}\right)+E_{2}^{n} F\left(\frac{i}{n^{k}}\right)+E_{3}^{n} F\left(\frac{i+1}{n^{k}}\right)+E_{2}^{n} F\left(\frac{i+2}{n^{k}}\right)+E_{1}^{n} F\left(\frac{i+3}{n^{k}}\right) .
$$

This further implies

$$
F\left(\frac{n i+n-1}{n^{k+1}}\right)=E_{1}^{n} F\left(\frac{-(i-1)}{n^{k}}\right)+E_{2}^{n} F\left(\frac{-i}{n^{k}}\right)+E_{3}^{n} F\left(\frac{-(i+1)}{n^{k}}\right)+E_{2}^{n} F\left(\frac{-(i+2)}{n^{k}}\right)+E_{1}^{n} F\left(\frac{-(i+3)}{n^{k}}\right) \text {. }
$$

Again implies

$$
\begin{aligned}
F\left(\frac{n i+n-1}{n^{k+1}}\right) & =E_{1}^{n} f_{-(i-1)}^{k}+E_{2}^{n} f_{-i}^{k}+E_{3}^{n} f_{-(i+1)}^{k}+E_{2}^{n} f_{-(i+2)}^{k}+E_{1}^{n} f_{-(i+3)}^{k} \\
& =f_{-n i+n-1}^{k+1}=F\left(-\frac{n i+n-1}{n^{k+1}}\right)
\end{aligned}
$$

which completes the proof.

In Figure 7, the basic limit functions of schemes (2.2) and (2.3) are shown. Here doted lines show the control polygons where the solid lines indicate the basic limit functions at different values of parameter. In 7 (a), limit functions are generated at $\mu=0.1,0.4$ and 0.68 from top to bottom, while in $7(\mathrm{~b})$, functions are generated at $\mu=0.15,0.45$ and 0.85 from top to bottom.

In Figure 8, we show performance of our schemes by setting different values of shape parameter. The control polygons are drawn by doted lines and the smooth curves by solid lines. Limit curves presented in 8 (a) are obtained by scheme $(2.2)$ at $\mu=0.15,0.45$, and 0.67 while limit curves shown in 8 (b) are obtained by scheme (2.3) at $\mu=0.2,0.45$, and 0.85 . These values are taken from parameter range for $\mathrm{C}^{1}$, $\mathrm{C}^{2}-$, and $\mathrm{C}^{3}$ - continuity of both schemes.

In Table 4, we compare some properties of proposed 5-point approximating subdivision schemes with some existing 5-point approximating schemes. It is clear from Table 4, the polynomial reproduction and approximation order of proposed schemes (2.2) and (2.3) are greater than the existing schemes [23, 25, 2831] while support size of proposed schemes (2.2) and (2.3) is smaller than these existing schemes. 


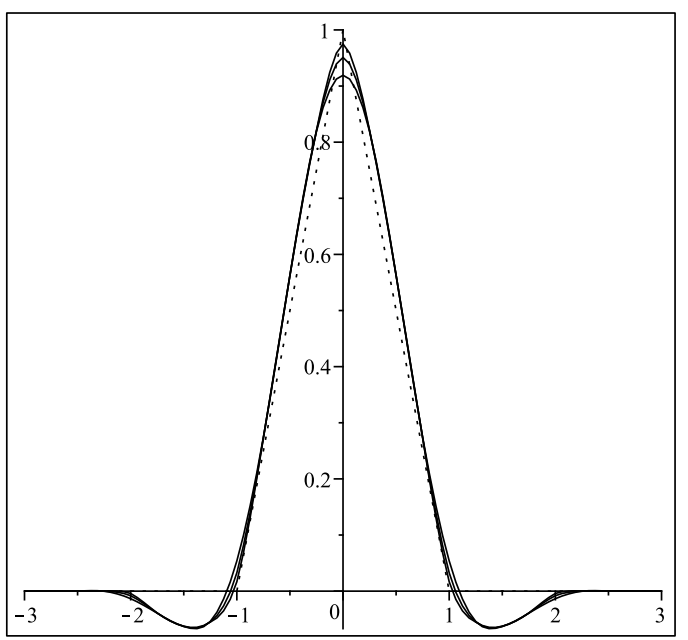

(a)

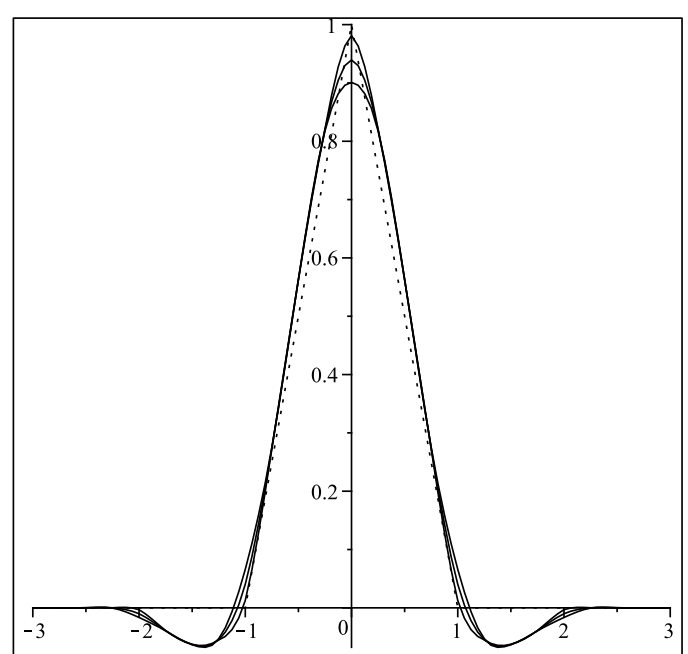

(b)

Figure 7: (a) and (b) show the effect of parameter on the shape of the basic limit function of the schemes (2.2) and (2.3), respectively.

Table 4: Comparison of proposed schemes with some existing schemes

\begin{tabular}{cccc}
\hline Scheme & $\begin{array}{c}\text { Polynomial } \\
\text { reproduction }\end{array}$ & Order & Support \\
\hline Binary 5-point [28] & 1 & 2 & 9 \\
Binary 5-point [23] & 1 & 2 & 9 \\
Binary 5-point [31] & 1 & 2 & 8 \\
Ternary 5-point [25] & 1 & 2 & 7 \\
Ternary 5-point [30] & 1 & 2 & 7 \\
Quaternary 5-point [29] & 1 & 2 & 6.333 \\
Quaternary 5-point [11] & 1 & 2 & 6.333 \\
Ternary 5-point proposed & 3 & 4 & 6 \\
Quaternary 5-point proposed & 3 & 4 & 5.333 \\
\hline
\end{tabular}

\section{Conclusion}

In this section, we give brief summary of work done so far. We present a simple and well-organized two-step algorithm which generates a class of 5-point n-ary approximating subdivision scheme. Shape preserving properties that is monotonicity, convexity, and concavity preservation of data fitting have been discussed. It is concluded that family of scheme has relaxed data fitting depending on the values of parameter.

We also present smoothness analysis of proposed scheme. Some important properties of proposed scheme like Hölder exponent, degree of polynomial generation, polynomial reproduction, approximation order support and symmetry of basic limit function have been discussed. An explicit formula to calculate support width of basic limit function is established. We deduced that arity and support width of 5-point n-ary scheme are reciprocal to each other. Also as we increase the arity of 5-point n-ary schemes, support of the schemes decreases and it is generally observed that as we decrease the arity of the scheme the Hölder exponent increases. For large arity of the scheme the Hölder exponent approaches to 1. Visual performance of proposed schemes are shown by several examples. 


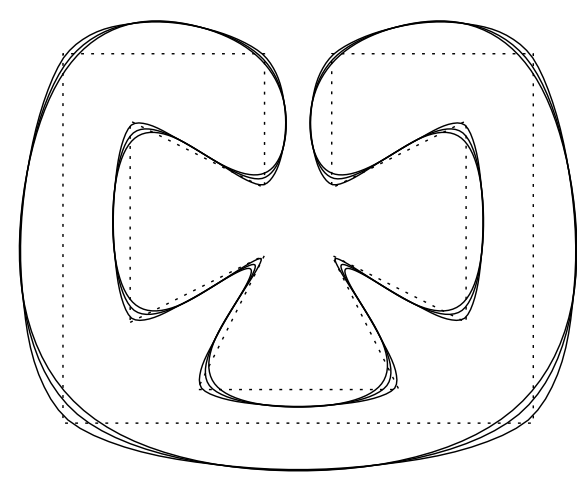

(a)

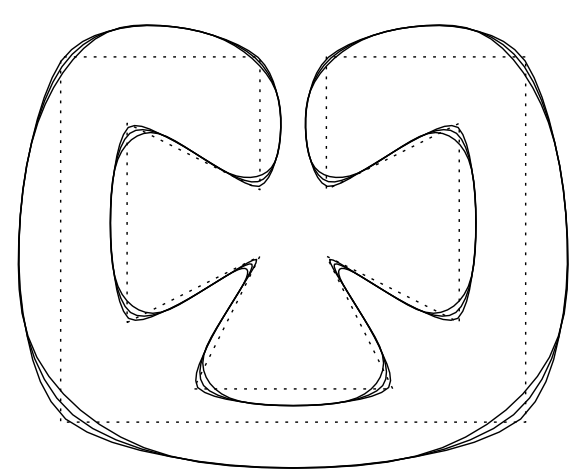

(b)

Figure 8: (a) and (b) show the effect of parameter on the shape of limit curves of the schemes (2.2) and (2.3), respectively.

\section{Acknowledgment}

This work is supported by the Indigenous Ph.D. Scholarship Scheme of Higher Education Commission (HEC) Pakistan and NRPU No-3183.

\section{References}

[1] U. H. Augsdöefer, N. A. Dodgson, M. A. Sabin, Variation on the four-point subdivision scheme, Comput. Aided Geom. Design, 27 (2010), 78-95. 1, 2.1

[2] Z. J. Cai, Convergence, error estimation and some properties of four-point interpolation subdivision scheme, Comput. Aided Geom. Design, 12 (1995), 459-468. 1

[3] Z. J. Cai, Convexity preservation of the interpolating four-point $\mathrm{C}^{2}$ ternary stationary subdivision scheme, Comput. Aided Geom. Design, 26 (2009), 560-565. 1, 3.11, 4

[4] G. M. Chaikin, An algorithm for high speed curve generation, Comp. Grap. Image Proces., 3 (1974), 346-349. 1

[5] C. Conti, K. Hormann, Polynomial reproduction for univariate subdivision scheme of any arity, Approx. Theory, 163 (2011), 413-437. 4

[6] G. De Rham, Un peu de mathmatiques propos d'une courbe plane, Elemente der Math, 2 (1947), 678-689. 1

[7] G. Deslauries, S. Dubuc, Symmetric iterative interpolation processes, Constr. Approx., 5 (1989), 49-68. 2, 2.1

[8] N. Dyn, F. Kuijt, D. Levin, R. van Damme, Convexity preservation of the four-point interpolatory subdivision scheme, Comput. Aided Geom. Design, 16 (1999), 789-792. 1, 3.11, 4

[9] N. Dyn, D. Levin, Subdivision scheme in the geometric modeling, Acta Numerica, 11 (2002), 73-144. 2.2, 2.1, 4

[10] N. Dyn, D. Levin, J. A. Gregory, A 4-point interpolatory subdivision scheme for curve design, Comput. Aided Geom. Design, 4 (1987), 257-268. 1

[11] A. Ghaffar, Lower and higher arity subdivision schemes and their applications, Ph.D. thesis, Department of Mathematics, Islamia university of Bahawalpur, (2013). 4

[12] A. Ghaffar, G. Mustafa, K. Qin, The 4-point a-ary approximating subdivision scheme, Open J. Appl. Sci., 3 (2013), 106-111. 1

[13] A. Ghaffar, G. Mustafa, K. Qin, Unification and application of 3-point approximating subdivision schemes of varying arity, Open J. Appl. Sci., 2 (2012), 48-52. 1

[14] Y.-X. Hao, R.-H. Wang, C.-J. Li, Analysis of a 6-point binary subdivision scheme, Appl. Math. Comput., 218 (2011), 3209-3216. 1, 3.11, 4

[15] M. F. Hassan, I. P. Ivrissimitzis, N. A. Dodgson, M. A. Sabin, An interpolating 4-point $C^{2}$ ternary stationary subdivision scheme, Comput. Aided Geom. Design, 19 (2002), 1-18. 1

[16] M. Z. Hussan, S. Bashir, Shape preserving surface data visualization using rational bi-cubic functions, J. Numer. Math., 19 (2011), 267-307. 3.10, 2, 3

[17] M. Z. Hussan, M. Sarfraz, T. S. Shaikh, Monotone data visualization using rational functions, World Appl. Sci. J., 16 (2012), 1496-1508. 3.1, 3.10

[18] J. M. Lane, R. F. Riesenfeld, A theoretical development for computer generation and display of piecewise polynomial surfaces, IEEE Trans. Patt. Anal. Mach. Intel., 2 (1980), 35-46. 1 
[19] A. L. Mehaute, F. I. Uteras, Convexity-preserving interpolatory subdivision, Comput. Aided Geom. Design, 11 (1994), 17-37. 3.4, 3.7

[20] G. Mustafa, J. Deng, P. Ashraf, N. A. Rehman, The mask of odd points n-ary interpolating subdivision scheme, J. Appl. Math., 2012 (2012), 20 pages. 1

[21] G. Mustafa, L. Hao, J. Zhang, J. Deng, $l^{1}$-regression based subdivision schemes for noisy data, Computer-Aided Design., 58 (2015), 189-99. 4

[22] G. Mustafa, F. Khan, A new 4-point quaternary approximating subdivision scheme, Abstr. Appl. Anal., 2009 (2009), 14 pages. 1

[23] G. Mustafa, F. Khan, A. Ghaffar, The m-point approximating subdivision scheme, Lobachevskii J. Math., 30 (2009), 138-145. 4, 4

[24] G. Mustafa, N. A. Rehman, The mask of $(2 b+4)$-point $n$-ary subdivision scheme, Computing, 90 (2010), 1-14. 1

[25] F. Pitolli, Ternary shape-preserving subdivision schemes, Math. Comput. Simulation, 106 (2014), 185-194. 4, 4

[26] O. Rioul, Simple regularity for subdivision schemes, SIAM J. Math. Anal., 23 (1992), 1544-1576. 4

[27] S. Samreen, Shape preserving data visualization with rational quatric splines, M.Phil. thesis, Department of Mathematics, University of the Punjab, (2006). 3.11

[28] S. S. Siddiqi, N. Ahmad, A new five-point approximating subdivision scheme, Int. J. Comput. Math., 85 (2008), 65-72. 4,4

[29] S. S. Siddiqi, M. Younis, The m-point quaternary approximating subdivision schemes, Amer. J. Comput. Math., 3 (2013), 6-10. 4

[30] S. S. Siddiqi, M. Younis, Construction of ternary approximating subdivision schemes, Politehn. Univ. Bucharest Sci. Bull. Ser. A Appl. Math. Phys., 76 (2014), 71-78. 4

[31] J. Tan, X. Zhuang, L. Zhang, A new four-point shape-preserving $C^{3}$ subdivision scheme, Comput. Aided Geom. Design, 31 (2014), 57-62. 1, 3.10, 3.11, 2, 4, 4, 4 